\title{
A support group created a caring environment where women with heart disease felt understood, supported, and strengthened by peers and nurse facilitators
}

Arthur HM, Wright DM, Smith KM. Women and heart disease: the treatment may end but the suffering continues. Can J Nurs Res 2001;33:17-29.

\section{QUESTION: From the perspectives of women with heart disease, what is the apparent benefit of a community based communication and psychoeducational support group?}

Design

Participatory action research.

Setting

Hamilton, Ontario, Canada.

Participants

16 women (46-76 y) who had experienced a myocardial infarction, coronary artery bypass graft surgery, or percutaneous transluminal coronary angioplasty in the previous 6 months or angina in the previous year, and who had no physical or mental health disabilities.

\section{Methods}

Through a process of discussion, deliberation, and evaluation, participants codesigned a community based communication and psychoeducational support group for themselves that they thought would be beneficial to other women living with heart disease. 2 groups of women ( $\mathrm{n}=9$ and $\mathrm{n}=7$ ) met monthly in 2 hour sessions for 5 months in a local church. A nurse clinician and a nurse researcher jointly facilitated sessions. Women participated in a telephone support network and were asked to keep a diary of their experiences of living with heart disease. Consensus was used to choose the focus of the sessions. Sessions were videotaped and recorded using field notes, and data were transcribed. Analysis used the constant comparison method and reflexive critique.

\section{Main findings}

The main finding was that participants' expressions of suffering and loss were facilitated both by the relationships among the women and between the women and the nurse facilitators. Women described the recovery period as "terrible". They felt isolated, vulnerable, and confused, and suppressed their emotions within their families and with friends. Family and friends seemed less likely to understand their feelings of pain than were their support group peers. The group setting appeared to be more suitable for expressing feelings, helped to relieve anxiety, and helped bring suppressed emotions to the surface. The group setting created a caring environment where women felt heard, understood, supported, and strengthened.

"Keeping up appearances" seemed to be part of women's relationships with their families and physicians. At home, women felt the need to appear to be engaged in normal family routines. With physicians, women were not given opportunities to express their suffering and could not comfortably mourn their experiences of loss.

Women felt that the expert facilitators were essential to

Source of funding: Hamilton Community Foundation.

For correspondence: Dr H Arthur, School of Nursing, McMaster University, Hamilton, Ontario, Canada. arthurh@momaster.ca the promotion of caring and revelation of suffering. The facilitators were seen as providing information resources and a comforting and understanding presence. In contrast, the women had mixed feelings about the telephone support network. Although they appreciated the concept, they were reluctant to interfere with other women's lives and did not want to appear "selfish" and "needy".

\section{Conclusion}

For women living with heart disease, a professionally run support group created an environment for caring, revelation, and alleviation of suffering. In this setting, women felt heard, understood, supported, and strengthened by both their support group peers and the nurse facilitators.

\section{COMMENTARY}

The aim of the study by Aruthur et al was to develop and implement a community based communication and psychoeducational support group for women living with heart disease. Using a participatory action research (PAR) approach, Arthur et al, together with the participants, focused on articulating the informational and emotional needs of women living with heart disease.

The authors are to be commended for creating an awareness of the contextual complexity of the social constructions of how women live with heart disease. This research has revealed that the issues reported for women recovering from cardiac events are different from those reported for men. Researchers often overlook the needs of women living with long term illness. ${ }^{1}$ Few inquiries have focused on women's perspectives. Only relatively recently has it been acknowledged that the findings of research on men living with chronic illness may not be relevant to women because of the social construction of gender. ${ }^{2}$

The study by Arthur et al offers health professionals new knowledge that can be applied when caring for women recovering from cardiac events. The process and method of the study were clearly described and the findings provide readers with a sensitive insight to the longitudinal nature of women's recovery from cardiac events. It seems important for health professionals to provide women living with heart disease the opportunity to openly communicate their concerns and experiences on an ongoing basis. The findings of this study remind healthcare professionals that they should be guided by the needs of individual women rather than simply advocating prescriptive disease education. Hence, the findings are of importance to medical and nursing clinicians, but also the wider healthcare community.

The PAR process can be liberating, empowering, and educative, and can facilitate validation of participant knowledge and experiences. The acknowledgment, affirmation, and validation that women experience in a group setting seem profoundly important to ongoing recovery. The articulation of the PAR process used in this inquiry may be useful for organisations considering the development of similar programmes.

Debbie Kralik, RN, PhD Research Associate Research Unit, Royal District Nursing Service Glenside, South Australia, Australia

1 Thorne S, McCormick J, Carty E. Deconstructing the gender neutrality of chronic illness and disability. Health Care Women 997;18:1-16.

Kralik D, Koch T, Webb C. The domination of chronic illness research by biomedical interests. Aus J Holist Nurs 2001;8:4-12. 